\title{
Los lantánidos: ni tierras ni raras
}

\author{
다) Fernando Echeverri L.*, (1) John Jairo Parra B. \\ Universidad de Antioquia, Instituto de Química, Medellín, Colombia
}

\begin{abstract}
Resumen
La tabla periódica de los elementos contiene la base fundamental del conocimiento pasado, presente y futuro de la Química. Sin embargo, aún se proponen modelos adicionales y complementarios para tratar de satisfacer todas y cada una de las propiedades físicas y químicas, en especial de aquellos elementos que actualmente no se ajustan a ella. Algunas de esas desviaciones seguramente tendrán explicaciones por aproximaciones cuánticas, obviamente desconocidas en el tiempo de la propuesta original. Específicamente, existen dos situaciones relevantes clásicas, relacionadas con las tierras raras y los actínidos, que involucran unos 30 elementos. Estos se muestran como anexos por fuera del orden previsto y, por sus características de grupos, se organizan como períodos. A pesar de los debates en torno a la reubicación de dichos elementos, que realmente no es un mero ejercicio académico, las tierras raras son valiosos materiales con múltiples aplicaciones en la vida diaria y, además, tienen un papel fundamental en el equilibrio económico y político mundial. En este artículo se presentan varios aspectos relacionados con su posición en la tabla periódica, así como las propiedades estructurales y electrónicas de sus favorables y anómalos comportamientos, sus aplicaciones y el contexto internacional por la hegemonía mundial basada en el uso de sus elementos. (c) 2019. Acad. Colomb. Cienc. Ex. Fis. Nat.
\end{abstract}

Palabras clave: Lantánidos; Tierras raras; Configuración; Orbital $f$; Tabla periódica; Reasignación de la posición; Materiales estratégicos; Aplicaciones; Economía y política; China Vs. Estados Unidos.

The lanthanides: neither earths nor rare

\begin{abstract}
The periodic table of elements contains the fundamental basis of past, present, and future knowledge of Chemistry. However, additional and complementary models are still proposed to try to satisfy the unique physical and chemical properties of those that do not conform to it. Some of these questions will surely have explanations based on quantum approaches, obviously unknown in the time of the original proposal. In the periodic table, there are two typical relevant situations related to rare lands and actinides, involving about 30 elements. These are displayed as annexes outside the intended order and having group characteristics, they are organized as periods. Despite discussions regarding the relocation of these elements, which is not an irrelevant academic exercise, rare earths are now valuable materials with multiple applications and they have a fundamental role in economic balance and global politics. This article presents several aspects related to their position in the periodic table, as well as the structural and electronic properties of their favorable and unusual behaviors, their applications, and the international context for global hegemony founded on this type of elements. C 2019. Acad. Colomb. Cienc. Ex. Fis. Nat.
\end{abstract}

Key words: Lanthanides; Rare earths; Configuration; forbital; Periodic table; Position reassignment; Strategy material; Application; Economy and politics; China vs USA.

\section{Introducción}

Están en todos partes y vienen en múltiples colores y tamaños, crean interrogantes en todo momento, hacen más gratas nuestras vidas, pero su obtención requiere de largos procesos. En el peor de los momentos, son motivo de agrias disputas entre dos rivales que antes compartían todo. Son los elementos de las tierras raras, los cuales han sido objeto de casi dos siglos de investigación mediante laboriosos procesos que hoy se consideran rudimentarios, y de técnicas analíticas muy limitadas; por ello su purificación y sus aplicaciones han sido un reto para el ingenio humano. Aún contienen propiedades por explorar que pueden mejorar la calidad de nuestra vida, o terminarla, o modularla, ya que recientemente se han constituido en una herramienta valiosa del equilibrio geopolítico y económico mundial. Se podría afirmar con certeza que las aplicaciones tecnológicas y científicas de las tierras raras han marcado un hito en los últimos dos decenios y que durante los próximos años sus nuevos usos incidirán de manera notable en el trasporte, la generación de energía y la computación, entre otros.

\footnotetext{
*Correspondencia:

Fernando Echeverri; fernando.echeverri@udea.edu.co

Recibido: 17 de junio de 2019

Aceptado: 28 de junio de 2019

Editor: Sonia Moreno
} 
¿Qué tienen de especial las tierras raras, porqué se debate su posición en la tabla periódica, cuáles son sus usos y por qué son tan importantes en el ámbito mundial? Estos interrogantes se abordan en este ensayo, pero para responderlos es necesario ubicarse en el contexto de la racionalidad de la tabla periódica, que justamente ahora está cumpliendo 150 años.

\section{La tabla periódica}

Algunos elementos químicos que hoy conocemos se originaron en los primeros tiempos del universo, en una secuencia de reacciones que permitió generar la serie completa desde el hidrógeno hasta el hierro, dándose posteriormente el fenómeno de la fisión nuclear. A partir del hierro, el elemento más estable en la naturaleza, se empieza a presentar la fusión nuclear, para crear casi todos los demás elementos.

La tabla periódica vigente es una ordenación de los elementos químicos (63) y fue propuesta por el ruso Dmitri Mendeléiev, el alemán Julius Lothar Meyer y el inglés William Odling en 1869 con base en las ideas del italiano Stanislao Cannizzaro, después de haber asistido al primer Congreso Internacional de Química Pura y Aplicada realizado en Alemania. Su primera formulación se hizo en función de los pesos atómicos. En 1871 el mismo Mendeléiev publica la segunda versión de su tabla y agrupa los elementos en columnas y grupos. En 1911, antes de que se descubrieran los protones en el núcleo atómico, el físico aficionado Antonius van den Broek propuso en la revista Nature, que el orden de los elementos en la tabla periódica dependía del número de cargas positivas del núcleo, o sea, el número atómico. En 1913 Henry Moseley logra probar esa hipótesis estudiando los espectros de rayos $\mathrm{X}$ de 50 elementos químicos.

Ahora bien, en la propuesta original hubo otro hallazgo importante y es que con dicho ordenamiento se generaban conjuntos de elementos que tenían propiedades químicas muy similares y que hoy se conocen como grupos, de los cuales hay 18 según la IUPAC. También se ha establecido un tercer descriptor de los elementos en la tabla, llamado el bloque, que hace relación a la ocupancia de orbitales por los electrones y toman el nombre de estos, por lo tanto, los bloques se denominan $s, p, d$ y $f$. Este aporte se debió al químico norteamericano Glenn Seaborg, quien murió en 1999 a los 86 años de edad.

Estos son algunos datos curiosos de la tabla: el bromo $(\mathrm{Br})$, el mercurio $(\mathrm{Hg})$ y el galio $(\mathrm{Ga})$ en condiciones normales son líquidos y el francio se vuelve líquido dependiendo de la temperatura ambiente; hay 11 gases, y varias decenas de otros elementos sintéticos, algunos con cortísimas vidas medias. El astato $(Z=85)$ es el segundo elemento más escaso en el planeta y emite una cantidad muy grande de radioactividad, por lo que, en vez de usarse con fines nucleares, se ha podido aprovechar en radioterapia en células cancerosas. El berkelio ( $\mathrm{Bk}, \mathrm{Z}=97)$, elemento químico radioactivo, es el más escaso del planeta, porque se genera por la descomposición del uranio enriquecido
(U-239), lo que deja solamente unos átomos de berkelio. Los nombres de los elementos responden a los de países, ciudades, científicos, procesos, mitología, color, apariencia, grado de dificultad del proceso de obtención, etc. Solo uno lleva el nombre de una mujer, el meitnerio, y otro es compartido, el curio. Y hay desviaciones importantes, pues, por ejemplo, el agua no debería ser líquida en su estado natural, sino gaseosa.

En la tabla de Mendeléiev quedaron los huecos para predecir la existencia de algunos elementos descubiertos poco después, como el galio (1875), el escandio (1879), el germanio (1887), y el tecnecio (1937). Su descubrimiento posterior fue el sello de garantía de la precisión y racionalidad de orden propuesto. Sin embargo, a pesar de su exactitud, aún tiene puntos en discusión y, por ello, continuamente se proponen diferentes modelos u ordenamientos (Rich \& Laing, 2011), entre ellos el helicoidal, el esférico, el tetraédrico (Stewart, 2018), así como 32 columnas o grupos en vez de las 18 existentes (Lemonick, 2019). Todo esto para tratar de acomodar las propiedades "anómalas" de algunos elementos en el esquema original.

\section{Tierras raras, ni tan raras ni tan escasas}

La denominación de tierras raras es algo confusa: el término "tierra", que parece un poco arcaico, proviene de la antigua denominación que se daba a lo que hoy se conoce como óxidos, y que correspondía a materiales con apariencia amorfa, insolubles en agua y que no cambiaban su estado físico con el calentamiento. Erróneamente se considera que la palabra "rara" se adoptó por su baja concentración en los materiales a partir de los cuales se purificaban, pero varios de ellos son más abundantes incluso que los metales preciosos o que el mismo cobre y el plomo. También cabe la acepción de que se trataba de elementos que se utilizaban muy raramente, pues para la época de su descubrimiento tenían poca utilidad práctica. Pero, ante todo, la palabra hace referencia a la dificultad para extraerlos y purificarlos, tanto la de aquellos materiales que los contenían en forma bruta, como la de los que tenían propiedades químicas muy similares, lo que entorpecía extremadamente su separación.

Las tierras raras corresponden a una serie de elementos que se encuentran en una posición especial dentro de la tabla periódica y cuya comprensión requiere un análisis más a fondo, desde una perspectiva no rigurosamente mecánicocuántica. Los electrones en los átomos se encuentran en la periferia del núcleo ocupando niveles; de ellos se conocen los niveles K, L, M, N, O, P y Q $(1,2,3,4,5,6$ y 7, en términos actuales), los que, a su vez, están constituidos por subniveles $(s, p, d, f)$, que pueden contener $1,3,5$ y 7 orbitales, respectivamente, pero cada uno de ellos puede alojar solamente hasta dos electrones. Sin embargo, hay dos limitantes para situarlos. La primera es que hay un número máximo de electrones en cada orbital, nivel y subnivel, y la segunda es que estos se llenan en orden de menor a mayor energía, independientemente de la secuencia de los orbitales. 
En otras palabras, es probable que se ocupen primero los orbitales externos antes que otros internos, como en el caso del potasio y del calcio. Ahora bien, a medida que se incrementa el número de electrones y se llenan los niveles más externos, comienzan a observarse las influencias de otros factores, explicables por aproximaciones cuánticas. En el caso del cobre, por ejemplo, es curioso que su distribución electrónica sea $1 s^{2} 2 s^{2} 2 p^{6} 3 s^{2} 3 p^{6} 4 s^{1} 3 d^{10}$ y no $\ldots 4 s^{2}$ $3 d^{9}$. Esto es muy importante en algunas propiedades de las tierras raras.

Casi todas las tierras raras se originaron en dos materiales provenientes de sendas minas suecas, una de ellas en Itterby, por lo que se le dio el nombre de iterbita, y la otra en Bastnäs, en la cual se encontró la cerita (American Chemical Society, 1999). Secuencialmente, de ellas fueron obteniéndose productos que se clasificaron inicialmente como elementos puros, pero, posteriormente, algunos de ellos demostraron ser una mezcla. De la iterbita se generaron el itrio, el terbio, el erbio, el tulio, el disprosio, el holmio, el iterbio y el lutecio, y de la cerita, el cerio, el lantano, el praseodimio, el neodimio, el gadolinio, el samario y el europio. El praseodimio solamente se obtuvo en 1947 y constituye un elemento radioactivo generado por la descomposición del uranio.

Las tierras raras no se encuentran en la naturaleza en forma libre, como otros elementos; se hallan en minerales muy específicos como la allanita, la loparita, la parisita, la apatita, la monazita y otros (Balaram, 2018). Durante la Segunda Guerra Mundial fueron contaminantes de los materiales fisionables necesarios para fabricar la bomba atómica; esos conocimientos de los procesos de separación son la base para producirlos a escala industrial actualmente, y se basan fundamentalmente en el intercambio iónico (American Chemical Society, 1999).

\section{La rara posición y agrupación de las tierras raras en la tabla periódica}

Según la International Union of Pure and Applied Chemistry (IUPAC), las tierras raras corresponden a quince elementos de los llamados lantánidos $(\mathrm{Z}=57$ a $\mathrm{Z}=71)$, además del escandio y el itrio. Ellos, a su vez, se han dividido en dos series según su masa molecular: los lantánidos pesados, o ítricos, que son de menor concentración e incluyen el europio, el gadolinio, el terbio, el disprosio, el holmio, el erbio, el tulio, el iterbio y el lutecio, y los ligeros o céricos, como el lantano, el cerio, el praseodimio, el neodimio, el prometio y el samario.

Dentro de la tabla periódica las tierras raras hacen parte del grupo 3 (que define sus propiedades químicas), el período 6 (ordenados de acuerdo con el número creciente de protones), y el bloque $f$, que indica la presencia de electrones en ese orbital. De esta manera, los elementos de los lantánidos se incluyen como un periodo de acuerdo con su número atómico creciente (secuencia de izquierda a derecha en la tabla), pero por sus propiedades químicas son más bien un grupo (secuencia de arriba abajo). Realmente no tuvieron una posición definida en la tabla hasta que Glenn Seaborg, por comodidad gráfica, propuso localizarlos como una extensión del período VI, pero fuera de la misma tabla, algo que también tuvo que hacerse con los Actínidos (Lemonick, 2019). Es curioso, además, que ni en la misma tabla de la IUPAC se ha definido si el lantano y el actinio, o el lutecio y el laurencio, son elementos del grupo 3 (figura 1) (IUPAC, 2018).

La distribución electrónica de las tierras raras corresponde a la formula general $4 f^{\mathrm{n}+1} 5 s^{2}, 5 p^{6}, 6 s^{2}$. La disponibilidad de los tres electrones de los subniveles $5 d$ y $6 s$ hace que todos ellos sean trivalentes, incluidos el escandio $(3 d 4 s)^{3}$, el itrio $(4 d 5 s)^{3}$ y el lantano $(5 d 6 s)^{3}$, pero estos últimos no tienen electrones en el orbital $f$. Esto representa una diferencia fundamental frente a los demás elementos, ya que el orbital $4 f$ se va llenando paulatinamente, desde 0 electrones en el lantano hasta 14 electrones en el lutecio, pero hay que tener en cuenta que se presentan algunas excepciones en las que los electrones ocupan el subnivel $5 \mathrm{~d}$ en vez de llenar el $4 \mathrm{f}$ por razones de energía y distancia entre el núcleo y el orbital (Ce y el Gd). Por otra parte, los electrones de este orbital no participan en la formación de enlaces y, por lo tanto, no afectan su comportamiento químico, pero son la causa principal de las anómalas propiedades físicas de los elementos de las tierras raras que se explotan comercialmente.

\section{¿Por qué son magnéticos y luminiscentes los elementos de las tierras raras?}

Las peculiaridades de los elementos de las tierras raras, que incluyen efectos luminiscentes o fosforescentes y magnéticos, se explican fundamentalmente por los electrones del orbital $4 f$. En este contexto, se debe recordar que el llenado de los subniveles se hace de manera secuencial y ordenada y que siempre se comienzan a llenar con electrones los orbitales menos energéticos, pero, además, los espines de los electrones se mantienen orientados paralelamente, que es la configuración más estable. El llenado de electrones es normal hasta que se llega al tercer nivel, pues a partir de allí comienzan a manifestarse los efectos del solapamiento de los orbitales $4 f$ con los electrones situados en $5 s$ y $5 p$. Esto, a su vez, causa un incremento en la carga nuclear, por lo cual el tamaño efectivo de los átomos se contrae hasta en un $20 \%$ dentro de la secuencia de lantano a lutecio, lo que se conoce como la llamada Contracción Radial o Lantánida (Voncken, 2016). De esta manera, en el caso extremo del gadolinio, que tiene la configuración [Xe] $4 f^{7} 5 d^{1} 6 s^{2}$, hay siete electrones no apareados que se comportan como pequeños magnetos que contribuyen en gran medida al momento magnético total de los metales de las tierras raras, creándose así magnetos permanentes. Estas propiedades son extensivas a otros metales cuando se producen aleaciones que contienen tierras raras. En el caso del escandio, el itrio y el lantano no hay electrones desapareados y, por lo tanto, carecen de magnetismo. 


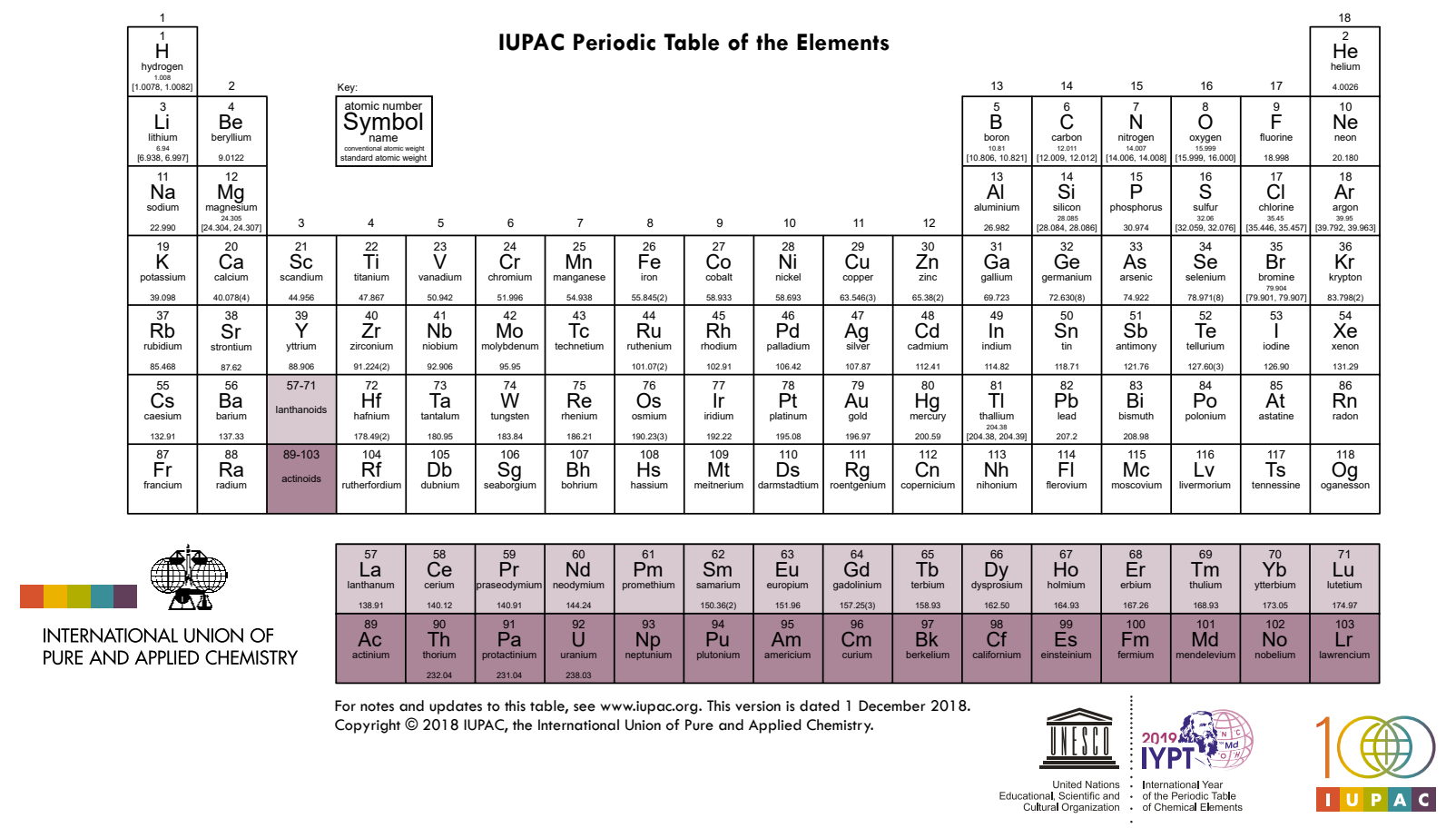

Figura 1. La tabla periódica según la IUPAC (IUPAC, 2018).

Los lantánidos presentan coloraciones verdes, rosadas y amarillas. Dichos colores son el resultado de la transición de un electrón entre diferentes orbitales $f$ existentes (transición $f-f)$. Esa diferencia es tan pequeña que basta la energía UV para excitarlos y generar luminiscencia por su efecto, lo que se aprovecha en el billete de 50 euros, al cual se añade una sal de europio que le da su florescencia característica cuando se observa bajo este tipo de luz (Ganguli \& Cook, 2018).

Por otra parte, y debido a lo anterior, la diferencia energética de los electrones de los orbitales $4 f$ y $5 d$ es tan pequeña que es fácil promover un electrón del orbital $f$ al $d$, generando la forma tetravalente del cerio, o bien, que un electrón del orbital $5 d$ sea transferido al $4 f$, con lo cual se explica el carácter divalente de metales como el samario, el europio y el iterbio (Voncken, 2016).

\section{Las tierras raras están en todas partes}

Si la vida moderna se midiera por la frecuencia de uso y la presencia de diversos materiales, indudablemente que los plásticos y los elementos de las tierras raras se llevarían el palmarés. No obstante, el empleo original de estos últimos es modesto, pues en sus inicios la utilidad más común era para fabricar piedras de encendedores.

Aunque algunas aplicaciones están aún en fase de experimentación, hoy son elementos tecnológicamente vitales y fuente de bienestar humano, y se emplean en las siguientes aplicaciones (Deacon \& Junk, 2014; Gschneidner \& Pecharsky, 2006; Voncken, 2016; Ritter, 2017; Balaram, 2018; Behrsing \& Kirkpatrick, 2019): catalizadores, baterías y motores de carros eléctricos, procesos petroquímicos, computadores, discos duros, lectores de $\mathrm{CD}$, tecnología nuclear, televisores y pantallas (color verde con terbio y rojo con europio), autenticidad de billetes, decoración, iluminación, turbinas eólicas, óptica y pulimiento de vidrio y cristales, lámparas LED, láseres, fibra óptica, tubos catódicos, resonancia magnética de imágenes para diagnóstico, micrófonos, audífonos y un largo etcétera que incluye también elementos para defensa y ataque (aviones, barcos, tanques, submarinos, radares). Se augura que pronto habrá carros eléctricos con motores sin ineficientes engranajes y refrigeración basada en aleaciones que no requieren de gases ni de compresores. Tampoco la alta costura se ha olvidado de ellos, con el reemplazo de botones plásticos por piezas de neodimio. El gadolinio es especialmente importante en aleaciones refrigerantes, así como en el diagnóstico mediante resonancia magnética nuclear por los incrementos en la intensidad del campo de los tejidos que lo contienen y que se administra antes del examen.

\section{Las tierras raras como fuente de poder}

Mas allá del poder energético generado por las tierras raras, estas hacen parte importante del equilibrio tecnológico, económico y político del mundo. En China se concentra más del $70 \%$ de la producción mundial, cifra que algunos ubican hasta en un $90 \%$, aunque tiene un poco más del $30 \%$ de las reservas mundiales (Balaram, 2018). Para 1987, el dirigente chino Deng Xiao Ping hizo la primera adver-tencia del potencial de las tierras raras: "Occidente tiene petróleo. China, las tierras raras." (Hornby \& Sanderson, 2019). Esto ha sido posible por razones especialmente interesantes, 
como, por ejemplo, una planificación tecnológica a muy largo plazo y la presión importante sobre la competencia, que cerró las minas ante los precios momentáneamente bajos del mercado. La reactivación de una industria de este tipo tampoco es un proceso sencillo, corto y barato, puesto que pueden transcurrir casi diez años a partir de la prospección para que una mina comience a producir (iNemi, 2014; Geological Society of London, 2011). Pero adicionalmente, hay un factor que se esgrime para imponer cuotas mundiales y manejar los precios; China aduce que esos procesos son altamente contaminantes y corrosivos, y realmente lo son, y que, por lo tanto, debe preservar la salud de sus ciudadanos y cuidar el medio ambiente, lo que implica regular la producción de las tierras raras. Es cierto que parte de la basura final de la purificación consiste en ácido sulfúrico, soda cáustica, fluoruros, sulfuros e ingentes cantidades de agua, así como elementos radioactivos, con lo cual se deben extremar las precauciones y disponer cuidadosamente de esos residuos, lo que encarece y dificulta los procesos. Tal vez por esas razones de seguridad para sus connacionales y respeto a su medio ambiente es que Australia procesaba su materia prima en Malasia, pero cambios políticos recientes han reversado los acuerdos existentes (Law, 2019), por lo que, además de las tierras raras, Australia deberá encargarse de la deposición final de los cientos de miles de toneladas de desechos que se generan en ese proceso, incluidos el torio y el uranio.

Además de China y Australia, países como Estados Unidos, Brasil y Vietnam tienen significativas reservas de tierras raras, pero su producción es baja. En Colombia se desconoce si se han hecho intentos por reconocer la presencia de esos valiosos metales. Por otro lado, tanto las tierras raras como otros metales estratégicamente valiosos podrían explicar parcialmente por qué algunas empresas optan por importar rocas, tierra y piedra molida a través de los llamados 'cobreductos', así como los metales puros producidos in situ.

Valga una aclaración final. No se pueden confundir las tierras raras con otro mineral que también está estrechamente involucrado en procesos tecnológicos actuales, como es el coltán. Este contiene principalmente niobio y tantalio, pero tampoco se conoce un inventario nacional de sus existencias.

En algunos círculos financieros se considera que la amenaza de un embargo de esos materiales estratégicos por parte de China no tendría éxito, pues si bien ellos son productores, en Occidente esos metales se procesan y regresan a ese país como componentes importantes de sus productos tecnológicos (Binnemans, et al., 2013). Sin embargo, pocos quieren correr un riesgo, como cuando en 2010 ese país embargó los envíos de tierras raras a Japón por una disputa territorial, afectando notablemente la producción de importantes empresas como Hitachi, Honda y Panasonic, multiplicando estratosféricamente su costo. Aunque hubo una reclamación ante la Organización Mundial del Comercio, solo se procedió a un fallo dos años después, cuando ya el daño económico estaba hecho.
El reciente embargo del presidente Trump a bienes de importación de China fue respondido por el presidente chino Xi Jinping con una visita a la ciudad de Ganzhou, (Stevenson, 2019), centro de producción de buena parte de las tierras raras, en una clara advertencia de su respuesta futura. Peor aún, cuando en China se procesaba la producción de Mountain Pass, la mina productora de tierras raras en Estados Unidos, ésta, que era prácticamente la única representante norteamericana del ramo, quebró en el 2002. Posteriormente, en el 2015 reactivó su producción, pero para el 2018 fue rematada al mejor postor por un precio irrisorio. No sobra mencionar que su principal comprador fue una empresa china (Kirkpatrick, 2019; Topf, 2017).

Por otra parte, estos vaivenes políticos han traído consigo una inestabilidad económica que multiplica el precio del kilogramo de tierras raras en 10 a 20 veces. Por esta razón, las iniciativas privadas de exploración y explotación tienen un alto riesgo, y solo factores de seguridad nacional, aunados a subsidios, podrían reactivar el negocio de las tierras raras en Occidente.

Visto que en una cadena de producción no se puede sustituir súbitamente un elemento químico por otro y que poner a funcionar una mina es tarea de casi un decenio, la opción más inmediata parece ser reciclar las tierras raras (Balaram, 2018; Binnemans, et al., 2013), lo que no es fácil, porque ya está comprobada la dificultad para separarlos entre sí. Por otra parte, también se exploran otras fuentes como las minas de fertilizantes de fosfatos y los residuos de la combustión del carbón y de procesos nucleares. Occidente no está preparado para soportar el embargo de unos metales que, aunque carecen de una posición definida en la tabla periódica, tienen un puesto notable en el equilibrio geopolítico mundial.

\section{Conclusiones}

A pesar de que la tabla periódica tiene indudable valor constitutivo y predictivo para la Química, aún queda por definirse la posición correcta de los elementos correspondientes a las tierras raras y los Actínidos. Es claro que situarlos como apéndices en una tabla bidimensional no satisface el ordenamiento básico propuesto por sus autores originales. Tal vez a través de la 'multidimensionalidad' y el empleo de parámetros cuánticos pueda llegarse a un modelo que incluya esos 30 elementos, que constituyen casi un $25 \%$ de los conocidos hasta ahora. Día tras día el ser humano hace ingentes esfuerzos por descubrir más elementos, obviamente a través de reactores nucleares o ciclotrones o aceleradores de partículas. Más allá de que su ubicación correcta pueda ser un ejercicio académico, con una tabla periódica homogénea y consistente existe la posibilidad de conocer más profundamente cómo se organiza la materia y de qué manera se pueden optimizar sus propiedades para el bienestar humano.

El aspecto científico tiene también un componente geopolítico. De manera muy simplista se puede afirmar que esos molestos electrones del orbital $f$, que tanto perturban 
la armonía actual de la tabla periódica, son responsables de otra revolución tecnológica en la cual, entre otras cosas, el quemar y desperdiciar reservas fósiles para obtener energía serán ventajosamente sustituidos por procesos más limpios y eficientes que emplean el magnetismo y otras propiedades particulares de los elementos que constituyen las tierras raras. Esto siempre y cuando el orden mundial no se desordene y prevalezca la armonía tecnológica entre quienes poseen el recurso y quienes tienen el conocimiento para explotarlo en bien del ser humano. De otra manera, podríamos ser testigos de esa fatal premonición atribuida a Einstein: la cuarta guerra mundial se hará con palos y piedras.

\section{Contribución de los autores}

Fernando Echeverri: idea y redacción. John Jairo Parra: revisión de la distribución electrónica y conceptos de la tabla periódica.

\section{Conflicto de intereses}

Los autores declaran que no tienen conflicto de intereses.

\section{Referencias}

American Chemical Society. (1999). Separation of rare earth elements. 1999. Fecha de consulta: 8 de junio de 2019. Disponible en: https:/www.acs.org/content/dam/acsorg/ education/whatischemistry/landmarks/earthelements/ separation-of-rare-earth-elements-commemorative-booklet.pdf

Balaram, V. (2018). Rare earth elements: A review of applications, occurrence, exploration, analysis, recycling, and environmental impact. Geoscience Frontiers. 10: 1285-1303. Doi: 10.1016/j.gsf.2018.12.005

Behrsing, T., Deacon, G.B., Junk, P.C. (2014) The chemistry of rare earth metals, compounds and corrosion inhibitors. En: Forsyth, Maria, and Hinton, Bruce (editores.) Rare Earthbased Corrosion Inhibitors. Woodhead Publishing Series in Metals and Surface Engineering (61). Elsevier, Amsterdam, the Netherlands, p. 1-37.

Binnemans, K., Jones, P.T., Blanpain B., Van Gerven, T., Yang, Y., Walton, A., Buchert, M. (2013). Recycling of rare earths: A critical review. Journal Cleaner Production. 51: 1-22.

Ganguli, R., \& Cook, D. (2018). Rare earths: A review of the landscape. MRS Energy \& Sustainability. 5: E9. Doi: 10.1557/ mre.2018.7
Gschneidner, K.A., Jr \& Pecharsky, V.K. (2006). Rare Earths and Magnetic Refrigeration. Journal of Rare Earths. 24: 641-647

Hornby, L. \& Sanderson, H. (2019). Rare earths: Beijing threatens a new front in the trade war. Financial Times, Jun 3.

IUPAC. (2018). Periodic Table of Elements. Fecha de consulta: 8 de junio de 2019. Disponible en: https://iupac.org/what-wedo/periodic-table-of-elements/

iNEMI. (2014). White Paper: Rare Earth Metals Current Status \& Future Outlook. iNEMI Rare Earth Metals Project Team Second Quarter. Fecha de consulta: 20 de junio de 2019. Disponible en: http://thor.inemi.org/webdownload/ projects/iNEMI_REM_Whitepaper.pdf

Geological Society of London. (2011). Rare Earth Elements. Fecha de consulta: 2 de junio de 2019. Disponible en: https://www.geolsoc.org.uk/ /media/shared/documents/ policy/Rare \%20Earth \%20Elements \%20briefing $\% 20$ note $\% 20$ final $\% 20 \% 20 \% 20$ new\%20format.pdf

Law, Y-H. (2019). Politics could upend global trade in rare earth elements. Science 364: 114-115. DOI: 10.1126/ science.364.6436.114

Lemonick, S. (2019). Rearranging the table. Chem Eng News. 97: 26-29.

Kirkpatrick. K. (2019). Electronics Need Rare Earths. Communications ACM. 62: 17-18. Doi: 10.1145/3303847

Rich, R.L. \& Laing. M. (2011). Can the periodic table be improved? Educ. Quím. 22 (2): 162-165.

Ritter, S.K. (2017). A whole new world for rare earths. Chem Eng News. 95: 30-34.

Stevenson, A. (2019). China Faces New 'Long March' as Trade War Intensifies, Xi Jinping Says. The New York Times, May 21. Fecha de consulta: 8 de junio de 2019. Disponible en: https://www.nytimes.com/2019/05/21/world/asia/xijinping-china-trade.html

Stewart, P.J. (2018). Tetrahedral and spherical representations of the periodic system. Foundations of Chemistry. 20: 111-120.

Topf, A. (2017). Mountain Pass sells for \$20.5 million. Mining. Com. Fecha de consulta: 20 de junio de 2019. Disponible en: https://www.mining.com/mountain-pass-sells-20-5-million

Voncken, J.H.L. (2016). The Rare Earth Elements, Springer Briefs in Earth Sciences, Dordrecht: Springer, 2016. 127 p. Doi: 10.1007/978-3-319-26809-5 3 